\title{
Detection of spontaneous pneumothorax with chest ultrasound in the emergency department
}

\author{
Alfredo Barillari · Sampsa Kiuru
}

Received: 14 October 2009/ Accepted: 4 December 2009/Published online: 2 February 2010

(C) SIMI 2010

\section{Introduction}

A 38-year-old man was brought by ambulance to the Emergency Department (ED) with left sided chest pain, which occurred while he was competing in a bicycle race. The pain increased with deep inspiration, and did not radiate. The patient denied shortness of breath, abnormal sweating, or palpitations.

The physical examination was unremarkable: the lung sounds were clear and symmetrical, the heart tones were regular without murmurs. The vital signs were: blood pressure $132 / 80 \mathrm{mmHg}$, heart rate 80 beats per minute, respiratory rate 16 breaths per minute, oxygen saturation while breathing room air $99 \%$, temperature $36.8^{\circ} \mathrm{C}$.

The patient had no family history of coronary artery disease, and did not smoke. He had sustained a spontaneous pneumothorax 20 years earlier. The ECG showed sinus rhythm without acute ST changes, therefore, making myocardial ischemia an unlikely differential diagnosis.

Chest ultrasound, performed at the bedside, showed absence of "lung sliding" at the left apex, absence of B lines and presence of A lines. On M-mode, the typical finding of the "stratosphere sign" confirmed the presence of a left apical pneumothorax.

Chest radiography confirmed the presence of the left pneumothorax. The right lung was normal on chest scan. Chest ultrasound findings of pneumothorax have a high sensitivity in ruling out pneumothorax, and in the supine patient, this tool is more reliable than a chest X-ray study.

\footnotetext{
A. Barillari $(\bowtie) \cdot S$. Kiuru

Emergency Department, Southland Hospital,

Kew Road, 9812 Invercargill, New Zealand

e-mail: alfredobarillari@gmail.com
}

"Lung point", not found in this patient, is a dynamic image containing both normal and pneumothorax findings which has a specificity of $100 \%$.

\section{Lung ultrasound: examination technique and findings}

The patient can be scanned in a supine or sitting position, over the anterior, lateral and posterior areas bilaterally. These areas are identified by the anterior and posterior axillary lines. Longitudinal and transverse scans are equally acceptable, provided that the underlying structures are correctly identified. Each area is scanned from the apex to the base. The average time to scan the chest is around $3 \mathrm{~min}$ for the experienced ED sonographer. Multiple probes can be used: multifrequence phased array (2.53.5 MHz), convex and microconvex $(3.5-7.0 \mathrm{MHz})$ and linear probes (5.0-10 MHz) (Fig. 1).

Lung sonography largely consists of the analysis of artifacts because only artifacts appear on the screen. However, the ribs can be identified because they produce a posterior shadow. In a longitudinal scan between two ribs and about $0.5 \mathrm{~cm}$ deeper, an horizontal hyperechoic line is produced by the pleural interface.

Other hyperechoic horizontal lines appearing below the pleural line and parallel to this, are normal artifacts of an aerated lung: these are called "A lines" (Fig. 2) [1].

At the pleural line, a dynamic sign can be described: "lung sliding". It is a basic sign of normality due to the movement of the visceral pleural on the parietal pleura. The amplitude of the lung sliding is minimal at the apices and maximal at the bases. Lung sliding can be objectified and documented with M-mode. The "sea-shore sign" refers to the image of lung sliding with M-mode: the outer motionless part of the chest wall generates horizontal 


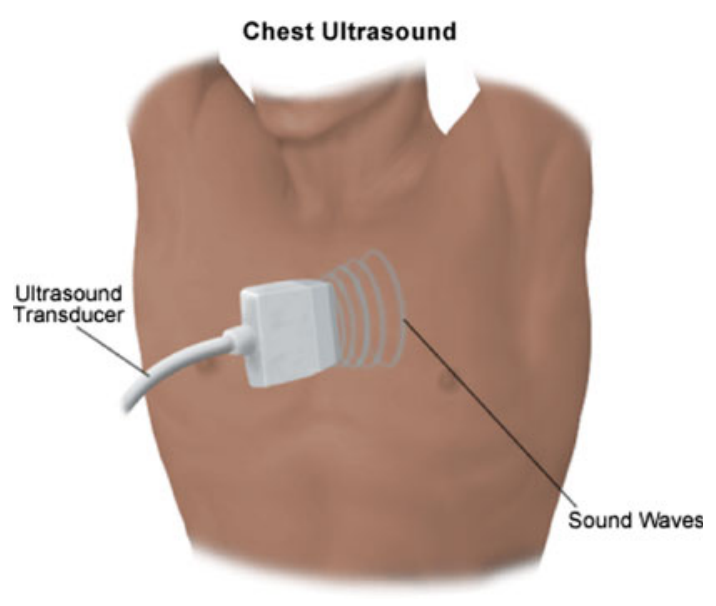

Fig. 1 Lung scanning technique

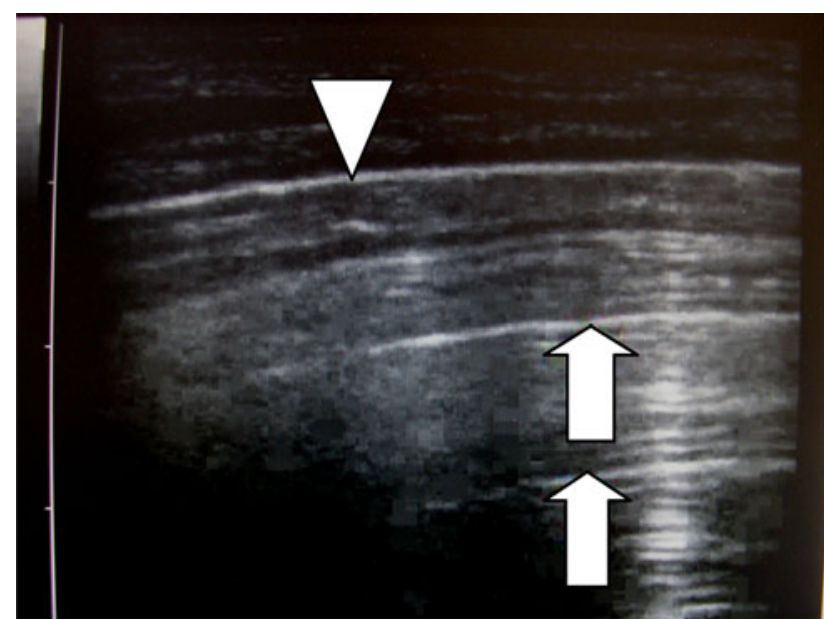

Fig. 2 A Lines (arrows) and pleural line (arrowhead)

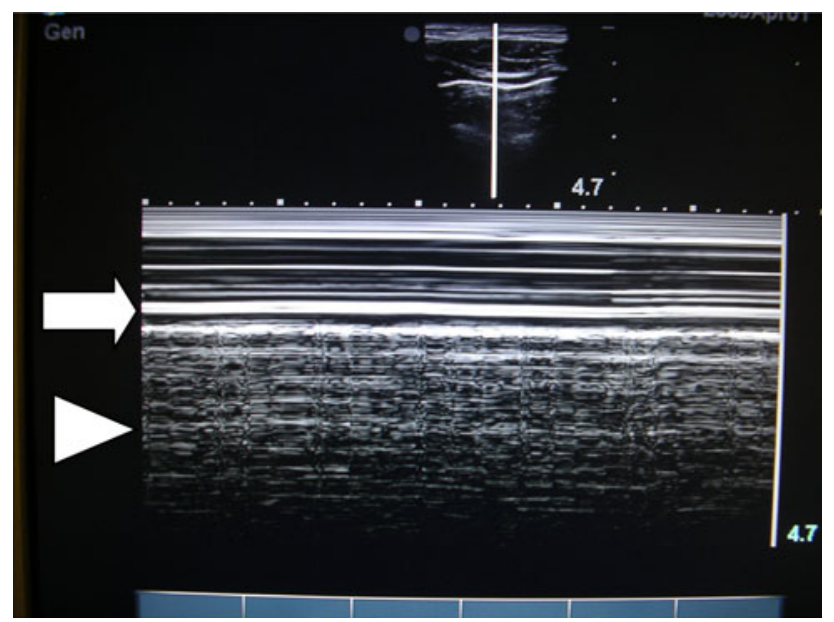

Fig. 3 Normal lung on M-mode scan:the pleural line (arrow) is an hyperechoic line on top of the screen. The subcutaneous tissues, from top of the screen to the pleural line appear as parallel horizontal lines (sea). The lung below the pleural line moves with respirations and therefore the horizontal lines disappear (arrowhead) with motion, resembling the sand (shore). This pattern of normality has been termed "sea-shore sign" lines-the waves, the deep motion artifacts below the pleural line generate the sandy pattern (Fig. 3). [2]

The "B lines" or "comet tails" arise from the pleural line, are well defined and laser-like beams, spreading to the lower edge of the screen, without fading and synchronous with lung sliding. They are generated by elements with a high-acoustic impedance gradient from the surrounding structures, such as fluid surrounded by air in the alveoli (Fig. 4) [3].

Pneumothorax contains only air and no fluid. It has a nondependent semiotic. Therefore, the anterior and lower areas should be scanned first in the supine patient [2]. Lung sliding should be sought. Absent lung sliding is a basic and initial step for the diagnosis. The abolition of lung sliding

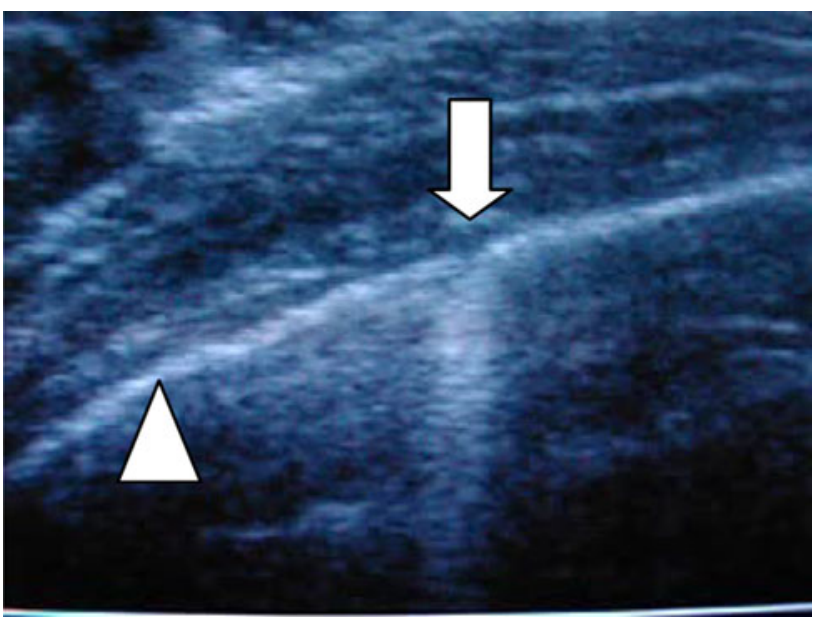

Fig. 4 B line (arrow), pleural line (arrowhead) B lines, or comet tails, are hyperechoic lines arising from the pleural line and reaching the bottom of the screen. They are perpendicular and synchronous with the movements of the pleural line

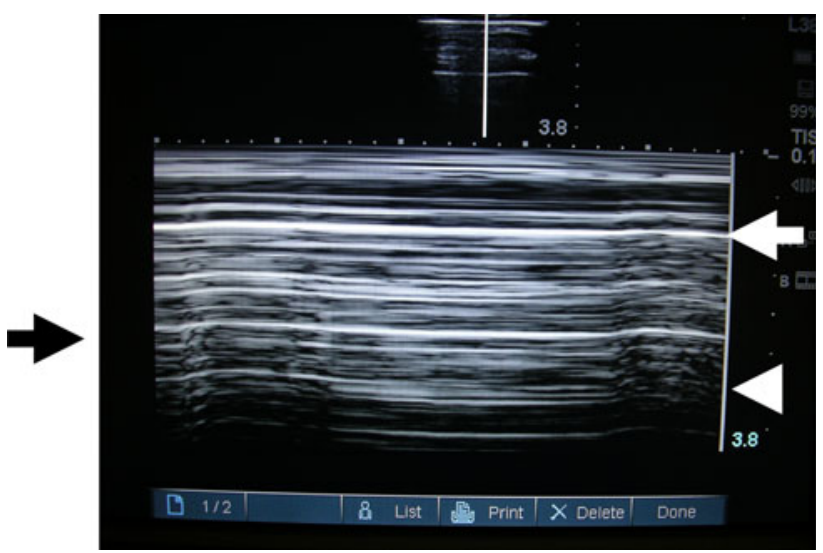

Fig. 5 Pneumothorax: the pleural line (white arrow) is an hyperechoic line on top of the screen. The "stratosphere sign" (white arrowhead) on M-mode is due to the absence of parenchymal lung movements and is shown as parallel horizontal lines. An A line is shown (black arrow): it is a thick hyperechoic line below and parallel to the pleural line 
can be objectified in M-mode, which gives a characteristic pattern, the "stratosphere sign" (Fig. 5).

\section{Discussion}

Lung ultrasound has a greater specificity than chest X-ray study in the detection of pneumothorax in the supine patient [2]. The lack of lung sliding, a finding of pneumothorax, shows only multiple A lines, an artifact due to the presence of air, that do not allow one to calculate the size of the pneumothorax. A small as well as a huge pneumothorax has the same ultrasound appearance. Therefore, chest X-ray study is mandatory to quantify the extent of the pneumothorax. Tension pneumothorax has no further ultrasound findings, and therefore is still a clinical diagnosis. The patient had a small apical pneumothorax, and no treatment was deemed necessary. The patient had no other studies.

Conflict of interest None.

\section{References}

1. Lichtenstein D, Goldstein I, Mourgeon E, Cluzel P et al (2004) Comparative diagnostic performances of auscultation, chest radiography, and lung ultrasonography in acute respiratory distress syndrome. Anesthesiology 100:9-15

2. Lichtenstein DA (2007) Ultrasound in the management of thoracic disease. Crit Care Med 35:S250-S261 (review)

3. Frassi F, Gargani L, Gligorova S, Ciampi Q et al (2007) Clinical and echocardiographic determinants of ultrasound lung comets. Eur J Echocardiogr 8:474-479 